\title{
A new generalization of Halanay-type inequality and its applications
}

Haiyang Wen', Shi Shu' and Liping Wen ${ }^{1 *}$ (D)

\section{*Correspondence:}

Ipwen@xtu.edu.cn

${ }^{1}$ School of Mathematics and

Computational Science, Xiangtan

University, Xiangtan, P.R. China

\begin{abstract}
In this paper, in order to study the dissipativity of nonlinear neutral functional differential equations, a generalization of the Halanay inequality is given. We apply this generalized Halanay inequality to an analysis of the dissipativity of two classes of nonlinear neutral delay integro-differential equations and the sufficient conditions are presented to ensure these systems are dissipative.
\end{abstract}

Keywords: Neutral delay integro-differential equations; Dynamical systems; Halanay inequality; Functional differential equations

\section{Introduction}

In 1966, in order to discuss the stability of the zero solution of the delay differential equation

$$
u^{\prime}(t)=A u(t)+B u(t-\tau), \quad \tau>0,
$$

Halanay introduced the following lemma (see [1] p. 378).

Lemma 1.1 (Basic Halanay inequality) Assume that $\tau \geq 0$ and $v(t)$ is a positive function defined on $\left[t_{0}-\tau,+\infty\right)$, with derivative $v^{\prime}(t)$ on $\left[t_{0},+\infty\right)$. If

$$
v^{\prime}(t) \leq-\alpha v(t)+\beta \sup _{t-\tau \leq s \leq t} v(s), \quad t \geq t_{0},
$$

where $\alpha>\beta>0$, then there exist $\gamma>0$ and $k>0$ such that

$$
v(t) \leq k \exp \left(-\gamma\left(t-t_{0}\right)\right), \quad t \geq t_{0} .
$$

The Halanay inequality became a powerful tool in the stability theory of delay differential equations, therefore many authors improved or generalized it to more general type and used it for investigating the stability and dissipativity of various functional differential equations. We refer the reader to the papers, for instance, of Baker and Tang [2], Agarwal, Kim and Sen [3, 4], Baker [5], Liz and Trofimchuk [6], Tian [7], Wen, Yu and Wang [8, 9], Liu et al. [10], Wang [11], Hien et al. [12], and Gan [13].

(c) The Author(s) 2018. This article is distributed under the terms of the Creative Commons Attribution 4.0 International License (http://creativecommons.org/licenses/by/4.0/), which permits unrestricted use, distribution, and reproduction in any medium, provided you give appropriate credit to the original author(s) and the source, provide a link to the Creative Commons license, and indicate if changes were made. 
On the other hand, many interesting problems in physics and engineering are modeled by dissipative dynamical systems. These systems are characterized by the property of possessing a bounded absorbing set, which all trajectories enter in a finite time and thereafter remain inside of. In the study of dissipative systems, this asymptotic behavior of the system is of interest and important (see [14]). In 1994, Humphries and Stuart [15] first studied the analytical and numerical dissipativity of initial value problems (IVPs) in ODEs. Hereafter, a number of results on the analytical and numerical dissipativity with respect to various types of differential equations are presented (such as found in [16-21]).

In this paper, we first present a more general Halanay-type inequality in Sect. 2. Then, in Sect. 3, we use this inequality to discuss the analytical dissipativity of two classes of nonlinear neutral delay integro-differential equations (NDIDEs) and some sufficient conditions which ensure the systems to be dissipative are given. Finally, the paper ends with a conclusion.

\section{The generalized Halanay inequality}

For simplicity of presentation, we denote $f^{\left[t_{1}, t_{2}\right]}:=\sup _{t_{1} \leq \xi \leq t_{2}} f(\xi)$ and $f^{\left[t_{1},+\infty\right)}:=\sup _{\xi \geq t_{1}} f(\xi)$ for a bounded function $f$.

Theorem 2.1 Assume that $\tau \geq 0$ and $u(t), w(t)$ are non-negative functions defined on $\left[t_{0}-\right.$ $\tau,+\infty)$, with derivative $u^{\prime}(t)$ on $\left[t_{0},+\infty\right)$. If

$$
\left\{\begin{array}{l}
u^{\prime}(t) \leq R_{1}(t)+A(t) u(t)+B(t) u^{[t-\tau, t]}+C(t) w(t)+D(t) w^{[t-\tau, t]}, \\
w(t) \leq R_{2}(t)+F(t) u(t)+G(t) u^{[t-\tau, t]}+H(t) w^{[t-\tau, t]}
\end{array}\right.
$$

for $t \geq t_{0}$ and

$$
w^{\left[t_{0}-\tau, t_{0}\right]} \leq \frac{r_{2}}{1-H_{0}}+\frac{F_{0}+G_{0}}{1-H_{0}} u^{\left[t_{0}-\tau, t_{0}\right]},
$$

and there exists a constant $\sigma>0$ such that

$$
A(t)+B(t)+(C(t)+D(t)) \frac{F_{0}+G_{0}}{1-H_{0}} \leq-\sigma, \quad \forall t \geq t_{0} .
$$

Then, for $t \geq t_{0}$, we have

$$
\left\{\begin{array}{l}
u(t) \leq \frac{\gamma^{*}}{\sigma}+\phi e^{-\mu^{*}\left(t-t_{0}\right)} \\
w(t) \leq \frac{\gamma_{2}}{1-H_{0}}+\frac{F_{0}+G_{0}}{1-H_{0}} \frac{\gamma^{*}}{\sigma}+\frac{F_{0}+G_{0} e^{\mu^{*} \tau}}{1-H_{0} e^{\mu^{*} \tau}} \phi e^{-\mu^{*}\left(t-t_{0}\right)}
\end{array}\right.
$$

where $R_{1}(t), R_{2}(t),-A(t), B(t), C(t), D(t), F(t), G(t), H(t)$ are non-negative, continuous and bounded functions defined on $\left[t_{0},+\infty\right)$;

$$
\left\{\begin{array}{llll}
F_{0}=F^{\left[t_{0},+\infty\right)}, & G_{0}=G^{\left[t_{0},+\infty\right)}, & C_{0}=C^{\left[t_{0},+\infty\right)}, & D_{0}=D^{\left[t_{0},+\infty\right)}, \\
H_{0}=H^{\left[t_{0},+\infty\right)}, & \gamma_{1}=R_{1}^{\left[t_{0},+\infty\right)}, & \gamma_{2}=R_{2}^{\left[t_{0},+\infty\right)}, & \phi=u^{\left[t_{0}-\tau, t_{0}\right]},
\end{array}\right.
$$

and $0<H(t) \leq H_{0}<1, \gamma^{*}=\gamma_{1}+\frac{C_{0}+D_{0}}{1-H_{0}} \gamma_{2}$. The constant $\mu^{*}>0$ is defined as

$$
\mu^{*}=\inf _{t \geq t_{0}}\left\{\mu(t): \mu(t)+A(t)+B(t) e^{\mu(t) \tau}+\left(C(t)+D(t) e^{\mu(t) \tau}\right) \frac{F_{0}+G_{0} e^{\mu(t) \tau}}{1-H_{0} e^{\mu(t) \tau}}=0\right\} .
$$


Specially, if $R_{1}(t)=R_{2}(t) \equiv 0,(2.4)$ degenerates into following form:

$$
\left\{\begin{array}{l}
u(t) \leq \phi e^{-\mu^{*}\left(t-t_{0}\right)} \\
w(t) \leq \frac{F_{0}+G_{0} e^{\mu^{*} \tau}}{1-H_{0} e^{\mu^{*} \tau}} \phi e^{-\mu^{*}\left(t-t_{0}\right)}
\end{array} \quad t \geq t_{0}\right.
$$

Proof First, if $\tau=0$, from the second formula of (2.1), we have

$$
w(t) \leq \frac{R_{2}(t)}{1-H(t)}+\frac{F(t)+G(t)}{1-H(t)} u(t)
$$

Substituting (2.6) into the first formula of (2.1) shows that (2.1) degenerates into a differential inequality

$$
u^{\prime}(t) \leq R(t)+\widetilde{A}(t) u(t), \quad t \geq t_{0}
$$

where

$$
\left\{\begin{array}{l}
R(t)=R_{1}(t)+(C(t)+D(t)) \frac{R_{2}(t)}{1-H(t)}, \\
\widetilde{A}(t)=A(t)+B(t)+(C(t)+D(t)) \frac{F(t)+G(t)}{1-H(t)} .
\end{array}\right.
$$

Noting the condition (2.3), it is can be proved that

$$
u(t) \leq \frac{\gamma^{*}}{\sigma}\left(1-\exp \left(\int_{t_{0}}^{t} \widetilde{A}(s) d s\right)\right)+u\left(t_{0}\right) \exp \left(\int_{t_{0}}^{t} \widetilde{A}(s) d s\right)
$$

The combination of this formula and (2.6) shows that (2.4) holds with

$$
\mu^{*}=\inf _{t \geq t_{0}}\left\{-A(t)-B(t)-(C(t)+D(t)) \frac{F(t)+G(t)}{1-H_{0}}\right\} .
$$

It is obvious that $\mu^{*}>0$ under the assumption (2.3).

In the following we assume that $\tau>0$. For any given $t \in\left[t_{0},+\infty\right)$, we define function $E(\mu)$ on $\left[0, \frac{1}{\tau} \ln \frac{1}{H_{0}}\right)$ by

$$
E(\mu):=\mu+A(t)+B(t) e^{\mu \tau}+\left(C(t)+D(t) e^{\mu \tau}\right) \frac{F_{0}+G_{0} e^{\mu \tau}}{1-H_{0} e^{\mu \tau}} .
$$

From (2.7) we can see that

$$
E(0)<0, \quad \lim _{\mu \rightarrow \frac{1}{\tau} \ln \frac{1}{H_{0}}-0} E(\mu)=+\infty, \quad E^{\prime}(\mu)>0 .
$$

Therefore, there exists a unique $\mu \in\left(0, \frac{1}{\tau} \ln \frac{1}{H_{0}}\right)$ such that

$$
\mu+A(t)+B(t) e^{\mu \tau}+\left(C(t)+D(t) e^{\mu \tau}\right) \frac{F_{0}+G_{0} e^{\mu \tau}}{1-H_{0} e^{\mu \tau}}=0
$$

which defines an implicit function $\mu(t)$ for $t \geq t_{0}$. It is obvious that $\mu^{*} \geq 0$. Now we prove that $\mu^{*}>0$. 
In fact, if this is not true. Let $\widetilde{H}_{0}$ satisfying $0<H_{0}<\widetilde{H}_{0}<1$ and let $0<\varepsilon_{1}<\min \left\{\frac{\sigma}{2}\right.$, $\left.-\frac{1}{\tau} \ln \widetilde{H}_{0}, \frac{1}{\tau} \ln \left(\frac{\sigma}{2 Q}+1\right)\right\}$, where

$$
Q=B_{0}+\frac{C_{0} \widetilde{H}_{0}\left(G_{0}+F_{0} H_{0}\right)+D_{0} \widetilde{H}_{0}\left(F_{0}+G_{0}\right)+D_{0} G_{0}\left(1-H_{0}\right)}{\left(\widetilde{H}_{0}-H_{0}\right)\left(1-H_{0}\right)},
$$

and $B_{0}=B^{\left[t_{0},+\infty\right)}$.

Then there would exist $t^{*} \geq t_{0}$ such that $\hat{\mu}:=\mu\left(t^{*}\right)<\varepsilon_{1}$ and

$$
\hat{\mu}+A\left(t^{*}\right)+B\left(t^{*}\right) e^{\hat{\mu} \tau}+\left(C\left(t^{*}\right)+D\left(t^{*}\right) e^{\hat{\mu} \tau}\right) \frac{F_{0}+G_{0} e^{\hat{\mu} \tau}}{1-H_{0} e^{\hat{\mu} \tau}}=0 .
$$

Substituting (2.3) into (2.9) gives

$$
\begin{aligned}
0 & =\hat{\mu}+A\left(t^{*}\right)+B\left(t^{*}\right) e^{\hat{\mu} \tau}+\left(C\left(t^{*}\right)+D\left(t^{*}\right) e^{\hat{\mu} \tau}\right) \frac{F_{0}+G_{0} e^{\hat{\mu} \tau}}{1-H_{0} e^{\hat{\mu} \tau}} \\
& \leq \varepsilon_{1}-\sigma+\left(B_{0}+\frac{\left.C_{0}\left(F_{0} H_{0}+G_{0}\right)+D_{0}\left(F_{0}+G_{0}\right)+D_{0} G_{0} e^{\varepsilon_{1} \tau}\left(1-H_{0}\right)\right)}{\left(1-H_{0} e^{\varepsilon_{1} \tau}\right)\left(1-H_{0}\right)}\right)\left(e^{\varepsilon_{1} \tau}-1\right) \\
& \leq \varepsilon_{1}-\sigma+Q\left(e^{\varepsilon_{1} \tau}-1\right)<\varepsilon_{1}-\sigma+\frac{\sigma}{2}<0,
\end{aligned}
$$

which is a contradiction.

In order to verify (2.4), we first show that, for any $\varepsilon>0$,

$$
\left\{\begin{array}{l}
u(t)<\frac{\gamma^{*}}{\sigma}+\varepsilon+\phi e^{-\mu^{*}\left(t-t_{0}\right)}, \\
w(t)<\frac{\gamma_{2}}{1-H_{0}}+\frac{F_{0}+G_{0}}{1-H_{0}}\left(\frac{\gamma^{*}}{\sigma}+\varepsilon\right)+\frac{F_{0}+G_{0} e^{\mu \tau}}{1-H_{0} e^{\mu \tau}} \phi e^{-\mu^{*}\left(t-t_{0}\right)}, \quad t \geq t_{0} .
\end{array}\right.
$$

In fact, when $t=t_{0},(2.10)$ is evident by using (2.2).

If we suppose (2.10) is not true for $t>t_{0}$, then there would exist some $\varepsilon_{0}>0$ and $\varsigma>t_{0}$ such that when $t<\varsigma$

$$
\left\{\begin{array}{l}
u(t)<\frac{\gamma^{*}}{\sigma}+\varepsilon_{0}+\phi e^{-\mu^{*}\left(t-t_{0}\right)} \\
w(t)<\frac{\gamma_{2}}{1-H_{0}}+\frac{G_{0}+F_{0}}{1-H_{0}}\left(\frac{\gamma^{*}}{\sigma}+\varepsilon_{0}\right)+\frac{F_{0}+G_{0} \mu^{\mu^{*} \tau}}{1-H_{0} e^{\mu^{*} \tau}} \phi e^{-\mu^{*}\left(t-t_{0}\right)},
\end{array}\right.
$$

while when $t=\varsigma$, at least one of the following two equalities is true:

$$
u(\varsigma)=\frac{\gamma^{*}}{\sigma}+\varepsilon_{0}+\phi e^{-\mu^{*}\left(\varsigma-t_{0}\right)}
$$

and

$$
w(\varsigma)=\frac{\gamma_{2}}{1-H_{0}}+\frac{G_{0}+F_{0}}{1-H_{0}}\left(\frac{\gamma^{*}}{\sigma}+\varepsilon_{0}\right)+\frac{F_{0}+G_{0} e^{\mu^{*} \tau}}{1-H_{0} e^{\mu^{*} \tau}} \phi e^{-\mu^{*}\left(\varsigma-t_{0}\right)} .
$$

However, from the second formula of (2.1), when $\varsigma-\tau \geq t_{0}$, we have

$$
w(\varsigma) \leq R_{2}(\varsigma)+F(\varsigma) u(\varsigma)+G(\varsigma) \sup _{\varsigma-\tau \leq \xi \leq \varsigma} u(\xi)+H(\varsigma) \sup _{\varsigma-\tau \leq \xi \leq \varsigma} w(\xi)
$$




$$
\begin{aligned}
< & R_{2}(\varsigma)+F(\varsigma)\left(\frac{\gamma^{*}}{\sigma}+\varepsilon_{0}+\phi e^{-\mu^{*}\left(\varsigma-t_{0}\right)}\right)+G(\varsigma)\left(\frac{\gamma^{*}}{\sigma}+\varepsilon_{0}+\phi e^{-\mu^{*}\left(\varsigma-\tau-t_{0}\right)}\right) \\
& +H(\varsigma)\left(\frac{\gamma_{2}}{1-H_{0}}+\frac{F_{0}+G_{0}}{1-H_{0}}\left(\frac{\gamma^{*}}{\sigma}+\varepsilon_{0}\right)+\frac{F_{0}+G_{0} e^{\mu^{*} \tau}}{\left.1-H_{0} e^{\mu^{*} \tau} \phi e^{-\mu^{*}\left(\varsigma-\tau-t_{0}\right)}\right)}\right. \\
= & R_{2}(\varsigma)+H(\varsigma) \frac{\gamma_{2}}{1-H_{0}}+\left(F(\varsigma)+G(\varsigma)+H(\varsigma) \frac{F_{0}+G_{0}}{1-H_{0}}\right)\left(\frac{\gamma}{\sigma}+\varepsilon_{0}\right) \\
& +\left(F(\varsigma)+G(\varsigma) e^{\mu^{*} \tau}+\frac{F_{0}+G_{0} e^{\mu^{*} \tau}}{1-H_{0} e^{\mu^{*} \tau}} H_{0} e^{\mu^{*} \tau}\right) \phi e^{-\mu^{*}\left(\varsigma-t_{0}\right)} \\
\leq & \frac{\gamma_{2}}{1-H_{0}}+\frac{F_{0}+G_{0}}{1-H_{0}}\left(\frac{\gamma^{*}}{\sigma}+\varepsilon_{0}\right)+\frac{F_{0}+G_{0} e^{\mu^{*} \tau}}{1-H_{0} e^{\mu^{*} \tau}} \phi e^{-\mu^{*}\left(\varsigma-t_{0}\right)},
\end{aligned}
$$

and, when $\varsigma-\tau<t_{0}$, we have

$$
\begin{aligned}
w(\varsigma) \leq & R_{2}(\varsigma)+F(\varsigma) u(\varsigma)+G(\varsigma) \max \left\{\sup _{t_{0}-\tau \leq \xi \leq t_{0}} u(\xi), \sup _{t_{0} \leq \xi \leq \varsigma} u(\xi)\right\} \\
& +H(\varsigma) \max \left\{\sup _{t_{0}-\tau \leq \xi \leq t_{0}} w(\xi), \sup _{t_{0} \leq \xi \leq \varsigma} w(\xi)\right\} \\
< & R_{2}(\varsigma)+F(\varsigma)\left(\frac{\gamma^{*}}{\sigma}+\varepsilon_{0}+\phi e^{-\mu^{*}\left(\varsigma-t_{0}\right)}\right)+G(\varsigma)\left(\frac{\gamma^{*}}{\sigma}+\varepsilon_{0}+\phi\right) \\
& +H(\varsigma)\left(\frac{\gamma_{2}}{1-H_{0}}+\frac{F_{0}+G_{0}}{1-H_{0}}\left(\frac{\gamma^{*}}{\sigma}+\varepsilon_{0}\right)+\frac{F_{0}+G_{0} e^{\mu^{*} \tau}}{\left.1-H_{0} e^{\mu^{*} \tau} \phi\right)}\right. \\
\leq & \frac{\gamma_{2}}{1-H_{0}}+\frac{F_{0}+G_{0}}{1-H_{0}}\left(\frac{\gamma^{*}}{\sigma}+\varepsilon_{0}\right)+\frac{F_{0}+G_{0} e^{\mu^{*} \tau}}{1-H_{0} e^{\mu^{*} \tau}} \phi e^{-\mu^{*}\left(\varsigma-t_{0}\right)} .
\end{aligned}
$$

Hence (2.14) and (2.15) show that (2.13) is not true. Therefore we need only consider the case that (2.12) holds and we shall obtain a contradiction. Set

$$
v(t)=\frac{\gamma^{*}}{\sigma}+\varepsilon_{0}+\phi e^{-\mu^{*}\left(t-t_{0}\right)}, \quad z(t)=v(t)-u(t)
$$

Then $z(t)>0$ for $t<\varsigma$ and $z(\varsigma)=0$ and $z^{\prime}(\varsigma) \leq 0$. Hence from the first formula of (2.1) we have

$$
\begin{aligned}
z^{\prime}(\varsigma)= & v^{\prime}(\varsigma)-u^{\prime}(\varsigma) \\
\geq & -\phi \mu^{*} e^{-\mu^{*}\left(\varsigma-t_{0}\right)} \\
& -\left(R_{1}(\varsigma)+A(\varsigma) u(\varsigma)+B(\varsigma) u^{[\varsigma-\tau, \varsigma]}+C(\varsigma) w(\varsigma)+D(\varsigma) w^{[\varsigma-\tau, \varsigma]}\right) .
\end{aligned}
$$

If $\varsigma-\tau \geq t_{0}$, it follows from (2.11), (2.12), (2.16) and the definition of $\gamma^{*}$ that

$$
\begin{aligned}
z^{\prime}(\varsigma) \geq & -\gamma^{*}-\left(\frac{\gamma^{*}}{\sigma}+\varepsilon_{0}\right)\left(A(\varsigma)+B(\varsigma)+(C(\varsigma)+D(\varsigma)) \frac{F_{0}+G_{0}}{1-H_{0}}\right) \\
& -\phi e^{-\mu^{*}\left(\varsigma-t_{0}\right)} \\
& \times\left(\mu^{*}+A(\varsigma)+B(\varsigma) e^{\mu^{*} \tau}+\left(C(\varsigma)+D(\varsigma) e^{\mu^{*} \tau}\right) \frac{F_{0}+G_{0} e^{\mu^{*} \tau}}{1-H_{0} e^{\mu^{*} \tau}}\right) .
\end{aligned}
$$


From the definition of the function $\mu(t)$, we have

$$
\mu(\varsigma)+A(\varsigma)+B(\varsigma) e^{\mu(\varsigma) \tau}+\left(C(\varsigma)+D(\varsigma) e^{\mu(\varsigma) \tau}\right) \frac{F_{0}+G_{0} e^{\mu(\varsigma) \tau}}{1-H_{0} e^{\mu(\varsigma) \tau}}=0
$$

Therefore, it is easy to see that

$$
\begin{aligned}
\mu^{*}+ & A(\varsigma)+B(\varsigma) e^{\mu^{*} \tau}+\left(C(\varsigma)+D(\varsigma) e^{\mu^{*} \tau}\right) \frac{F_{0}+G_{0} e^{\mu^{*} \tau}}{1-H_{0} e^{\mu^{*} \tau}} \\
= & \mu^{*}-\mu(\varsigma)+B(\varsigma)\left(e^{\mu^{*} \tau}-e^{\mu(\varsigma) \tau}\right)+C(\varsigma)\left(\frac{F_{0}+G_{0} e^{\mu^{*} \tau}}{1-H_{0} e^{\mu^{*} \tau}}-\frac{F_{0}+G_{0} e^{\mu(\varsigma) \tau}}{1-H_{0} e^{\mu(\varsigma) \tau}}\right) \\
& +D(\varsigma)\left(\frac{F_{0}+G_{0} e^{\mu^{*} \tau}}{1-H_{0} e^{\mu^{*} \tau}} e^{\mu^{*} \tau}-\frac{F_{0}+G_{0} e^{\mu(\varsigma) \tau}}{1-H_{0} e^{\mu(\varsigma) \tau}} e^{\mu(\varsigma) \tau}\right) \\
& \leq 0,
\end{aligned}
$$

which substituting into (2.17) and noting the condition (2.3), gives

$$
w^{\prime}(\varsigma)=v^{\prime}(\varsigma)-u^{\prime}(\varsigma) \geq \sigma \varepsilon_{0}>0
$$

If $\varsigma-\tau<t_{0}$, it follows from (2.16) that

$$
\begin{aligned}
z^{\prime}(\varsigma) \geq & -\phi \mu^{*} e^{-\mu^{*}\left(\varsigma-t_{0}\right)}-R_{1}(\varsigma)-A(\varsigma) u(\varsigma)-B(\varsigma) \max \left\{\phi, u^{\left[t_{0}, \varsigma\right]}\right\} \\
& -C(\varsigma) w(\varsigma)-D(\varsigma) \max \left\{w^{\left[t_{0}-\tau, t_{0}\right]}, w^{\left[t_{0}, \varsigma\right]}\right\} .
\end{aligned}
$$

Thus we also can get (2.18) by simple derivation. This is in contradiction with our result $w^{\prime}(\varsigma) \leq 0$. Therefore the inequality (2.10) must hold for any given $\varepsilon>0$. Since $\varepsilon>0$ is arbitrary, we let $\varepsilon \rightarrow 0$ and obtain (2.4), which completes the proof of Theorem 2.1.

Remark 2.2 If $R_{1}(t)=R_{2}(t)=C(t)=F(t) \equiv 0$, we can obtain expression (2.5). Particularly, if we further assume that $C(t)=F(t) \equiv 0$, then (2.5) degenerates into a conclusion which is present in [11].

\section{Dissipativity of two classes of nonlinear neutral functional differential equations}

In this section, we consider several simple applications of Theorem 2.1 to the study of dissipativity for two classes of nonlinear neutral functional differential equations.

Let $X$ be a real or complex, finite-dimensional or infinite-dimensional Hilbert space with the inner product $\langle\cdot, \cdot\rangle$ and the corresponding norm $\|\cdot\|$.

\subsection{Dissipativity of nonlinear neutral delay integro-differential equations (NNDIDEs)}

Consider the IVPs in NNDIDEs as follows:

$$
\left\{\begin{array}{l}
y^{\prime}(t)=f\left(t, y(t), y(t-\tau), y^{\prime}(t-\tau), \int_{t-\tau}^{t} g(t, \xi, y(\xi)) d \xi\right), \quad t \geq t_{0} \\
y(t)=\phi(t), \quad y^{\prime}(t)=\phi^{\prime}(t), \quad t_{0}-\tau \leq t \leq t_{0}
\end{array}\right.
$$


where $\tau$ are positive constant, the functions $f:\left[t_{0},+\infty\right) \times X \times X \times X \times X \rightarrow X, g$ : $\left[t_{0},+\infty\right) \times\left[t_{0}-\tau,+\infty\right) \times X \rightarrow X, \phi:\left[t_{0}-\tau, t_{0}\right] \rightarrow X$ are assumed to be continuous functions and for any $t \geq t_{0}, y, u, v, w \in X, f$ and $g$ satisfy the conditions:

$$
\left\{\begin{array}{l}
2 \operatorname{Re}\langle f(t, y, u, v, w), y\rangle \leq \alpha\|y\|^{2}+\beta\|f(t, 0, u, v, w)\|^{2}, \\
\|f(t, y, u, v, w)\|^{2} \leq \gamma_{1}+L_{y}\|y\|^{2}+\omega\|f(t, 0, u, v, w)\|^{2}, \\
\|f(t, 0, u, v, w)\|^{2} \leq \gamma_{2}+L_{u}\|u\|^{2}+L_{v}\|v\|^{2}+L_{w}\|w\|^{2},
\end{array}\right.
$$

and

$$
\|g(t, \xi, u)\| \leq \lambda\|u\|, \quad t-\tau \leq \xi \leq t, t \geq t_{0},
$$

where $-\alpha, \beta, \gamma_{1}, \gamma_{2}, \omega, \lambda, L_{y}, L_{u}, L_{v}, L_{w}$ are all non-negative real constants.

Theorem 3.1 Let problem (3.1) satisfy (3.2) and (3.3) with $L_{v} \omega<1$, and initial value function $\phi(t)$ satisfy

$$
\max _{t_{0}-\tau \leq t \leq t_{0}}\left\|\phi^{\prime}(t)\right\|^{2} \leq \frac{\gamma_{1}+\omega \gamma_{2}}{1-\omega L_{v}}+\frac{L_{y}+\omega\left(L_{u}+\lambda^{2} \tau^{2} L_{w}\right)}{1-\omega L_{v}} \max _{t_{0}-\tau \leq t \leq t_{0}}\|\phi(t)\|^{2}
$$

Let $y(t)$ be the solution of (3.1). Assume that there exists a constant $\sigma>0$ such that

$$
\alpha+\frac{\beta\left(L_{u}+L_{v} L_{y}+\lambda^{2} \tau^{2} L_{w}\right)}{1-L_{v} \omega} \leq-\sigma
$$

Then

(1) for any $t \geq t_{0}$ we have

$$
\left\{\begin{aligned}
\|y(t)\|^{2} \leq & \frac{\beta\left(\gamma_{2}+L_{\nu} \gamma_{1}\right)}{\left(1-L_{v}\right) \sigma}+\phi_{0} e^{-\mu^{*}\left(t-t_{0}\right)} \\
\left\|y^{\prime}(t)\right\|^{2} \leq & \frac{\gamma_{1}+\omega \gamma_{2}}{1-\omega L_{\nu}}+\frac{L_{y}+\omega\left(L_{u}+\lambda^{2} \tau^{2} L_{w}\right)}{1-\omega L_{\nu}} \frac{\beta\left(\gamma_{2}+L_{\nu} \gamma_{1}\right)}{\left(1-L_{\nu} \omega\right) \sigma} \\
& +\frac{L_{y}+\omega\left(L_{u}+\lambda^{2} \tau^{2} L_{w}\right) e^{\mu^{*} \tau}}{1-\omega L_{\nu} e^{\mu^{*} \tau}} \phi_{0} e^{-\mu^{*}\left(t-t_{0}\right)}
\end{aligned}\right.
$$

where $\phi_{0}=\max _{t_{0}-\tau \leq t \leq t_{0}}\|\phi(t)\|^{2}, \mu^{*}>0$ is given as follows:

$$
\begin{aligned}
\mu^{*}= & \inf _{t \geq t_{0}}\left\{\mu(t): \mu(t)+\alpha+\beta\left(L_{u}+\lambda^{2} \tau^{2} L_{w}\right) e^{\mu(t) \tau}\right. \\
& \left.+\frac{L_{y}+\omega\left(L_{u}+L_{w} \lambda^{2} \tau^{2}\right) e^{\mu(t) \tau}}{1-\omega L_{v} e^{\mu(t) \tau}} \beta L_{\nu} e^{\mu(t) \tau}=0\right\} .
\end{aligned}
$$

(2) the system is dissipative, for any $\varepsilon>0$ the open ball

$$
B=B\left(0, \sqrt{\frac{\beta\left(\gamma_{2}+L_{v} \gamma_{1}\right)}{\left(1-L_{\nu} \omega\right) \sigma}+\varepsilon}\right)
$$

is an absorbing set. 
Proof Let

$$
\left\{\begin{array}{l}
u(t)=\|y(t)\|^{2}, \\
w(t)=\left\|y^{\prime}(t)\right\|^{2},
\end{array} \quad t \geq t_{0}-\tau\right.
$$

and

$$
z(t)=\int_{t-\tau}^{t} g(t, \xi, y(\xi)) d \xi, \quad t \geq t_{0} .
$$

Then when $t \geq t_{0}$, from (3.2) we have

$$
\begin{aligned}
u^{\prime}(t) & =\frac{d}{d t}\langle y(t), y(t)\rangle \\
& =2 \operatorname{Re}\left\langle y(t), f\left(t, y(t), y(t-\tau), y^{\prime}(t-\tau), z(t)\right)\right\rangle \\
& \leq \alpha u(t)+\beta\left\|f\left(t, 0, y(t-\tau), y^{\prime}(t-\tau), z(t)\right)\right\|^{2} \\
& \leq \alpha u(t)+\beta\left(\gamma_{2}+L_{u} u(t-\tau)+L_{v} w(t-\tau)+L_{w}\|z(t)\|^{2}\right) .
\end{aligned}
$$

Noting (3.3) one obtains

$$
\begin{aligned}
\|z(t)\| & \leq \lambda \int_{t-\tau}^{t}\|y(\xi)\| d \xi \\
& \leq \lambda \tau \max _{t-\tau \leq \xi \leq t}\|y(\xi)\|,
\end{aligned}
$$

which gives

$$
\|z(t)\|^{2} \leq \lambda^{2} \tau^{2} \max _{t-\tau \leq \xi \leq t} u(\xi)
$$

Substituting (3.9) into (3.8), we have

$$
u^{\prime}(t) \leq \beta \gamma_{2}+\alpha u(t)+\beta\left(L_{u}+L_{w} \lambda^{2} \tau^{2}\right) u^{[t-\tau, t]}+\beta L_{v} w(t-\tau) .
$$

On the other hand, from the second formula of (3.2) and (3.9) we have

$$
\begin{aligned}
w(t) & =\left\|f\left(t, y(t), y(t-\tau), y^{\prime}(t-\tau), z(t)\right)\right\|^{2} \\
& \leq \gamma_{1}+L_{y} u(t)+\omega\left(\gamma_{2}+L_{u} u(t-\tau)+L_{v} w(t-\tau)+L_{w}\|z(t)\|^{2}\right) \\
& \leq \gamma_{1}+\omega \gamma_{2}+L_{y} u(t)+\omega L_{v} w(t-\tau)+\omega\left(L_{u}+L_{w} \lambda^{2} \tau^{2}\right) u^{[t-\tau, t]} .
\end{aligned}
$$

Therefore, combining of (3.10) and (3.11), for $t \geq t_{0}$ we have

$$
\left\{\begin{array}{l}
u^{\prime}(t) \leq \beta \gamma_{2}+\alpha u(t)+\beta\left(L_{u}+L_{w} \lambda^{2} \tau^{2}\right) u^{[t-\tau, t]}+\beta L_{\nu} w^{[t-\tau, t]}, \\
w(t) \leq \gamma_{1}+\omega \gamma_{2}+L_{y} u(t)+\omega\left(L_{u}+L_{w} \lambda^{2} \tau^{2}\right) u^{[t-\tau, t]}+\omega L_{v} w^{[t-\tau, t]} .
\end{array}\right.
$$


Let

$$
\left\{\begin{array}{l}
R_{1}=\beta \gamma_{2}, \quad A=\alpha, \quad B=\beta\left(L_{u}+L_{w} \lambda^{2} \tau^{2}\right), \quad C=0, \quad D=\beta L_{v}, \\
R_{2}=\gamma_{1}+\omega \gamma_{2}, \quad F=L_{y}, \quad G=\omega\left(L_{u}+L_{w} \lambda^{2} \tau^{2}\right), \quad H=\omega L_{v} .
\end{array}\right.
$$

From Theorem 2.1 we can obtain (3.5) immediately. This completes the proof of Theorem 3.1 .

\subsection{Dissipativity of nonlinear neutral Volterra integro-differential equations (NNVIDEs)}

Consider the IVPs in NNVIDEs as follows:

$$
\left\{\begin{array}{l}
y^{\prime}(t)=f\left(t, y(t), y(t-\tau), \int_{t-\tau}^{t} K\left(t, s, y(s), y^{\prime}(s)\right) d s\right), \quad t \geq t_{0}, \\
y(t)=\phi(t), \quad y^{\prime}(t)=\phi^{\prime}(t), \quad t \in\left[t_{0}-\tau, t_{0}\right],
\end{array}\right.
$$

where $\tau>0$ is constant, $\phi$ is a continuous function, and the functions $f:\left[t_{0},+\infty\right) \times X \times$ $X \times X \rightarrow X$ and $K:\left[t_{0},+\infty\right) \times\left[t_{0}-\tau,+\infty\right) \times X \times X \rightarrow X$ satisfy the conditions for any $t \geq t_{0}, y, u, v \in X:$

$$
\left\{\begin{array}{l}
2 \operatorname{Re}\langle f(t, y, u, v), y\rangle \leq \gamma+\alpha\|y\|^{2}+\beta_{1}\|f(t, 0, u, v)\|^{2} \\
\|f(t, y, u, v)\|^{2} \leq L_{y}\|y\|^{2}+\beta_{2}\|f(t, 0, u, v)\|^{2} \\
\|f(t, 0, u, v)\|^{2} \leq L_{u}\|u\|^{2}+L_{v}\|v\|^{2} \\
\|K(t, s, u, v)\| \leq \mu\|u\|+L_{k}\|v\|, \quad(t, s) \in D
\end{array}\right.
$$

where $D=\left\{(t, s): t \in\left[t_{0},+\infty\right), s \in[t-\tau, t]\right\}, \gamma, \beta_{1}, \beta_{2}, \mu, L_{y}, L_{u}, L_{v}$ are non-negative real constants and $\alpha \leq 0$.

Theorem 3.2 Assume that (3.13) satisfies (3.14) with $2 \beta_{2} \tau^{2} L_{v} L_{k}^{2}<1$, and initial value function $\phi(t)$ satisfies

$$
\max _{t_{0}-\tau \leq t \leq t_{0}}\left\|\phi^{\prime}(t)\right\|^{2} \leq \frac{L_{y}+\beta_{2}\left(L_{u}+2 \tau^{2} \mu^{2} L_{v}\right)}{1-2 \beta_{2} \tau^{2} L_{v} L_{k}^{2}} \max _{t_{0}-\tau \leq t \leq t_{0}}\|\phi(t)\|^{2}
$$

Assume there exists a constant $\sigma>0$ such that

$$
\alpha+\beta_{1} \frac{L_{u}+2 \tau^{2} L_{v}\left(L_{k}^{2} L_{y}+\mu^{2}\right)}{1-2 \beta_{2} \tau^{2} L_{v} L_{k}^{2}} \leq-\sigma
$$

Let $y(t)$ be the solution of (3.13). Then

(1) for any $t \geq t_{0}$ we have

$$
\left\{\begin{array}{l}
\|y(t)\|^{2} \leq \frac{\gamma}{\sigma}+\phi_{0} e^{-\mu^{*}\left(t-t_{0}\right)}, \\
\left\|y^{\prime}(t)\right\|^{2} \leq \frac{L_{y}+\beta_{2}\left(L_{u}+2 \tau^{2} \mu^{2} L_{v}\right)}{1-2 \beta_{2} \tau^{2} L_{v} L_{k}^{2}} \frac{\gamma}{\sigma}+\frac{L_{y}+\beta_{2}\left(L_{u}+2 \tau^{2} \mu^{2} L_{v}\right) e^{\mu^{*} \tau}}{1-2 \beta_{2} \tau^{2} L_{v} L_{k}^{2} e^{\mu^{*} \tau}} \phi_{0} e^{-\mu^{*}\left(t-t_{0}\right)},
\end{array}\right.
$$

where $\phi_{0}=\sup _{t_{0}-\tau \leq \xi \leq t_{0}}\|\phi(\xi)\|^{2}, \mu^{*}>0$ is defined as

$$
\mu^{*}=\inf _{t \geq t_{0}}\left\{\mu(t): \mu(t)+\alpha+\frac{L_{u}+2 \tau^{2} L_{\nu}\left(L_{k}^{2} L_{y}+\mu^{2}\right)}{1-2 \beta_{2} \tau^{2} L_{v} L_{k}^{2} e^{\mu(t) \tau}} \beta_{1} e^{\mu(t) \tau}=0\right\} .
$$


(2) the system is dissipative, for any $\varepsilon>0$ the open ball

$$
\mathbf{B}=\mathbf{B}\left(0, \sqrt{\frac{\gamma}{\sigma}+\varepsilon}\right)
$$

is an absorbing set.

Proof Let

$$
\left\{\begin{array}{l}
u(t)=\|y(t)\|^{2}, \\
w(t)=\left\|y^{\prime}(t)\right\|^{2},
\end{array} \quad t \geq t_{0}-\tau\right.
$$

and

$$
\left.z(t)=\int_{t-\tau}^{t} K\left(t, s, y(s), y^{\prime}(s)\right) d s\right), \quad t \geq t_{0}
$$

From (3.14) we can obtain

$$
\begin{aligned}
\|z(t)\|^{2} & \leq \tau^{2}\left(\mu \max _{t-\tau \leq \xi \leq t}\|y(\xi)\|+L_{k} \max _{t-\tau \leq \xi \leq t}\left\|y^{\prime}(\xi)\right\|\right)^{2} \\
& \leq 2 \tau^{2}\left(\mu^{2} u^{[t-\tau, t]}+L_{k}^{2} w^{[t-\tau, t]}\right)
\end{aligned}
$$

and

$$
\begin{aligned}
u^{\prime}(t) & =2 \operatorname{Re}(f(t, y(t), y(t-\tau), z(t)), y(t)) \\
& \leq \gamma+\alpha u(t)+\beta_{1}\|f(t, 0, y(t-\tau), z(t))\|^{2} \\
& \leq \gamma+\alpha u(t)+\beta_{1}\left(L_{u} u(t-\tau)+L_{v}\|z(t)\|^{2}\right) \\
& \leq \gamma+\alpha u(t)+\beta_{1}\left(L_{u}+2 \mu^{2} \tau^{2} L_{v}\right) u^{[t-\tau, t]}+2 \beta_{1} \tau^{2} L_{v} L_{k}^{2} w^{[t-\tau, t]}
\end{aligned}
$$

and

$$
\begin{aligned}
w(t) & =\|f(t, y(t), y(t-\tau), z(t))\|^{2} \\
& \leq L_{y}\|y(t)\|^{2}+\beta_{2}\|f(t, 0, y(t-\tau), z(t))\|^{2} \\
& \leq L_{y}\|y(t)\|^{2}+\beta_{2}\left[L_{u} u(t-\tau)+L_{v}\|z(t)\|^{2}\right] \\
& \leq L_{y} u(t)+\beta_{2}\left(L_{u}+2 L_{v} \tau^{2} \mu^{2}\right) u^{[t-\tau, t]}+2 \beta_{2} \tau^{2} L_{v} L_{k}^{2} w^{[t-\tau, t]} .
\end{aligned}
$$

It can be summarized from (3.16) and (3.17) that

$$
\left\{\begin{array}{l}
u^{\prime}(t) \leq \gamma+\alpha u(t)+\beta_{1}\left(L_{u}+2 \tau^{2} \mu^{2} L_{v}\right) u^{[t-\tau, t]}+2 \beta_{1} \tau^{2} L_{v} L_{k}^{2} w^{[t-\tau, t]} \\
w(t) \leq L_{y} u(t)+\beta_{2}\left(L_{u}+2 \tau^{2} \mu^{2} L_{v}\right) u^{[t-\tau, t]}+2 \beta_{2} \tau^{2} L_{v} L_{k}^{2} w^{[t-\tau, t]}
\end{array}\right.
$$

We denote

$$
\gamma_{1}=\gamma, \quad A=\alpha, \quad B=\beta_{1}\left(L_{u}+2 \tau^{2} \mu^{2} L_{v}\right), \quad C=0, \quad D=2 \beta_{1} \tau^{2} L_{v} L_{k}^{2},
$$




$$
\gamma_{2}=0, \quad F=L_{y}, \quad G=\beta_{2}\left(L_{u}+2 \tau^{2} \mu^{2} L_{v}\right), \quad H=2 \beta_{2} \tau^{2} L_{v} L_{k}^{2} .
$$

Then from Theorem 2.1 we can complete the proof of Theorem 3.2.

Remark 3.3 From a numerical point of view, it is important to study the potential of numerical methods in preserving the qualitative behavior of the analytical solutions. Therefore, the results of Theorem 3.1 and Theorem 3.2 presented in this paper, provide the theoretical foundation for analyzing the dissipativity of the numerical methods when they are applied to the underlying systems.

\section{Acknowledgements}

This work is supported by Hunan Key Laboratory for Computation and Simulation in Science and Engineering.

\section{Funding}

This work is supported by NSF of China (No. 11371302, 11571291).

\section{Competing interests}

The authors declare that they have no competing interests.

\section{Authors' contributions}

Three authors contributed equally to writing of this paper. Three authors read and approved the final manuscript.

\section{Publisher's Note}

Springer Nature remains neutral with regard to jurisdictional claims in published maps and institutional affiliations.

Received: 9 August 2018 Accepted: 29 October 2018 Published online: 06 November 2018

\section{References}

1. Halanay, A.: Differential Equations: Stability, Oscillations, Time Lags. Academic Press, New York (1966)

2. Baker, C.T.H., Tang, A.: Generalized Halanay inequalities for Volterra functional differential equations and discretized versions. In: Corduneanu, C., Sandberg, I.W. (eds.) Volterra Equations and Applications, Arlington, TX, 1996. Stability and Control: Theory and Applications, vol. 10, pp. 39-55. Gordon and Breach, Amsterdam (2000)

3. Agarwal, R.P., Kim, Y.H., Sen, S.K.: Advanced discrete Halanay-type inequalities: stability of difference equations. J. Inequal. Appl. 2009, 535849 (2009). https://doi.org/10.1155/2009/535849

4. Agarwal, R.P., Kim, Y.H., Sen, S.K.: New discrete Halanay inequalities: stability of difference equations. Commun. Appl. Anal. 12, 83-90 (2008)

5. Baker, C.T.H.: Development and application of Halanay-type theory: evolutionary differential and difference equations with time lag. J. Comput. Appl. Math. 234, 2663-2682 (2010)

6. Liz, E., Trofimchuk, S.: Existence and stability of almost periodic solutions for quasilinear delay systems and the Halanay inequality. J. Math. Anal. Appl. 248, 625-644 (2000)

7. Tian, H.J.: Numerical and analytic dissipativity of the $\theta$-method for delay differential equation with a bounded variable lag. Int. J. Bifurc. Chaos 14, 1839-1845 (2004)

8. Wen, L.P., Yu, Y.X., Wang, W.S.: Generalized Halanay inequalities for dissipativity of Volterra functional differential equations. J. Math. Anal. Appl. 347(1), 169-178 (2008)

9. Wen, L.P., Wang, W.S., Yu, Y.X.: Dissipativity and asymptotic stability of nonlinear neutral delay integro-differential equations. Nonlinear Anal., Theory Methods Appl. 72(3-4), 1746-1754 (2010)

10. Liu, B., Lu, W., Chen, T.: Generalized Halanay inequalities and their applications to neural networks with unbounded time-varying delays. IEEE Trans. Neural Netw. 22(9), 1508-1513 (2011)

11. Wang, W.S.: A generalized Halanay inequality for stability of nonlinear neutral functional differential equations. J. Inequal. Appl. 2010, Article ID 475019 (2010). https://doi.org/10.1155/2010/475019

12. Hien, L.V., Phat, V.N., Trinh, H.: New generalized Halanay inequalities with applications to stability of nonlinear non-autonomous time-delay systems. Nonlinear Dyn. 82, 563-575 (2015)

13. Gan, S.Q.: Dissipativity of $\theta$-methods for nonlinear Volterra delay-integro-differential equations. J. Comput. Appl. Math. 206(2), 898-907 (2007)

14. Temam, R.: Infinite-Dimensional Dynamical Systems in Mechanics and Physics. Springer Applied Mathematical Sciences Series, vol. 68. Springer, Berlin (1988)

15. Humphries, A.R., Stuart, A.M.: Runge-Kutta methods for dissipative and gradient dynamical systems. SIAM J. Numer Anal. 31, 1452-1485 (1994)

16. Humphries, A.R., Stuart, A.M.: Model problems in numerical stability theory for initial value problems. SIAM Rev. 36, 226-257 (1994)

17. Huang, C.M.: Dissipativity of Runge-Kutta methods for dynamical systems with delays. IMA J. Numer. Anal. 20 153-166 (2000)

18. Wen, L.P., Li, S.F.: Dissipativity of Volterra functional differential equations. J. Math. Anal. Appl. 324, 696-706 (2006)

19. Wen, L.P., Liao, Q.: Dissipativity of one-leg methods for a class of nonlinear functional-integro-differential equations. J. Comput. Appl. Math. 318, 26-37 (2017) 
20. Wang, W.S.: Uniform ultimate boundedness of numerical solutions to nonlinear neutral delay differential equations. J. Comput. Appl. Math. 309, 132-144 (2017)

21. Li, D.F., Zhang, C.J., Wang, W.S.: Long time behavior of non-Fickian delay reaction-diffusion equations. Nonlinear Anal., Real World Appl. 13(3), 1401-1415 (2012)

Submit your manuscript to a SpringerOpen ${ }^{\odot}$ journal and benefit from:

- Convenient online submission

- Rigorous peer review

- Open access: articles freely available online

- High visibility within the field

- Retaining the copyright to your article

Submit your next manuscript at $\gg$ springeropen.com 